DOI: $10.15593 / 2224-9877 / 2016.1 .08$

УДК 621.7.044

\author{
Е.В. Бабакова ${ }^{1}$, М.А. Химич ${ }^{1,2}$, \\ А.А. Сапрыкин ${ }^{1}$, Е.А. Ибрагимов ${ }^{1}$

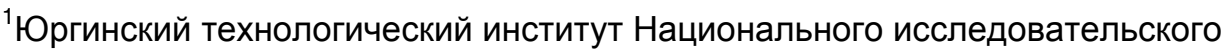 \\ Томского политехнического университета, Юрга, Россия \\ ${ }^{2}$ Институт физики прочности и материаловедения СО РАН, Томск, Россия
}

\author{
ПРИМЕНЕНИЕ СЕЛЕКТИВНОГО ЛАЗЕРНОГО \\ СПЛАВЛЕНИЯ ДЛЯ ПОЛУЧЕНИЯ НИЗКОМОДУЛЬНОГО \\ СПЛАВА СИСТЕМЫ ТИТАН - НИОБИЙ
}

\begin{abstract}
Сплав системы титан-ниобий широко используется при производстве имплантатов. В первую очередь это связано с низким модулем упругости и биоинертными свойствами сплава. Наибольшее распространение данные сплавы получили в зубном протезировании и ортопедии. На сегодняшний день сплав системы титан - ниобий в основном получают традиционными методами металлургии. При дальнейшем субтрактивном получении готового изделия образуется большое количество отходов. В связи с этим увеличивается стоимость конечного изделия. Противоположностью данным технологиям является аддитивное производство. Селективное лазерное сплавление (Selective Laser Melting) - технология, позволяющая печатать детали из порошков металлов и их смесей. Ее суть заключается в том, что слой материала в порошковой форме сплавляется лазером, затем поверх сплавленного слоя наносится следующий слой порошка и т.д. Технология используется для создания сложных изделий и рабочих прототипов. В данной работе рассмотрена возможность применения метода селективного лазерного сплавления для получения бинарного сплава из композитного порошка системы титан - ниобий. Получена панель образцов размером $10 \times 10$ мм при различных технологических параметрах. Образцы изготавливались на экспериментальной установке послойного лазерного синтеза «ВАРИСКАФ-100МВ». Данная установка позволяет управлять следующими технологическими параметрами: мощность лазерного излучения, скорость и шаг сканирования, температура подогрева порошка, толщина насыпаемого слоя, диаметр фокусировки пятна лазера. Все образцы получены в защитной среде аргона после предварительного вакуумирования. Построены зависимости изменения пористости и толщины сплавленного слоя от мощности лазерного излучения при разных скоростях сканирования. Установлено, что из всех контролируемых технологических параметров наибольшее влияние на формирование сплавленного слоя оказывает скорость сканирования и мощность лазерного излучения.
\end{abstract}

Ключевые слова: селективное лазерное сплавление, технологические параметры, металлические порошки, титан, ниобий, композитный порошок, скорость сканирования, стратегия сканирования, мощность лазерного излучения, сплавленный слой, пористость. 


\author{
E.V. Babakova ${ }^{1}$, M.A. Khimich, ${ }^{1,2}$, \\ A.A. Saprykin ${ }^{1}$, E.A. Ibragimov ${ }^{1}$ \\ ${ }^{1}$ Yurga Institute of Technology of National Research Tomsk \\ Polytechnic University Affiliate, Yurga, Russian Federation \\ ${ }^{2}$ Institute of Strength Physics and Materials Science of the Siberian Branch \\ of the Russian Academy of Sciences, Tomsk, Russian Federation
}

\title{
APPLICATION OF SELECTIVE LASER MELTING TO PRODUCE LOW MODULUS ALLOYS \\ OF THE TITANIUM-NIOBIUM
}

\begin{abstract}
The alloy system Ti-Nb widely used in the manufacture of implants. First of all this is due to a low elastic modulus and bioinert properties of the alloy. The most widespread of these alloys are obtained in dentistry and orthopedics. To date, the alloy system Ti-Nb is mainly produced by traditional metallurgy techniques. With further subtractive obtaining final product generates large quantities waste. In this regard, the increased cost of the final product. These technology is the opposite of additive manufacturing. Selective laser melting has a technology that allows to print parts made of metal powders and their mixtures. Its essence lies in that the layer of material in powder form is fused by the laser. Then, in the fused layer of powder is applied to the next layer and the process repeated. The technology is used to create complex products and working prototypes. In this paper, we consider the possibility of using selective laser melting to produce a binary alloy from a composite powder of $\mathrm{Ti}-\mathrm{Nb}$. The samples panel is obtained $10 \times 10 \mathrm{~mm}$ at different technological parameters. The samples were prepared by a layer by layer laser synthesis machine "VARISKAF-100 MV". It allows control the following process parameters: the laser power; speed and scanning step; the heating temperature of the powder; layer thickness; the diameter of the focus spot of the laser. All samples were prepared in a protective atmosphere of argon, after preliminary vacuumization. It were constructed dependences to the changes of porosity and thickness of the fused layer of the laser power at different scanning speeds. It was established that all of the controlled process parameters, the greatest influence on the formation of the fused layer has a scanning speed and laser power.
\end{abstract}

Keywords: selective laser melting, technological parameters, metal powders, titanium, niobium, composite powder, scanning speed, strategy scanning, laser power, fused layer, porosity.

Титан и его сплавы широко используются в качестве материалов для изготовления медицинских имплантатов и хирургических инструментов. Наибольшее распространение данные сплавы получили в зубном протезировании и ортопедии (рис. 1). Это связано с хорошими механическими свойствами, биосовместимостью и высокой коррозионной стойкостью сплавов.

Одним из недостатков титана и сплавов на его основе является высокий модуль упругости (более 100 ГПа). В связи с этим основная часть механической нагрузки распределяется на кости, а не на имплантат, что приводит к резорбции костной ткани (рис. 2), ослабле- 
нию имплантата и повторной операции [2]. В настоящее время ведутся поиск и разработка сплавов, имеющих низкий модуль упругости, при этом сохраняющих высокую прочность и хорошую пластичность изделия [3, 4].
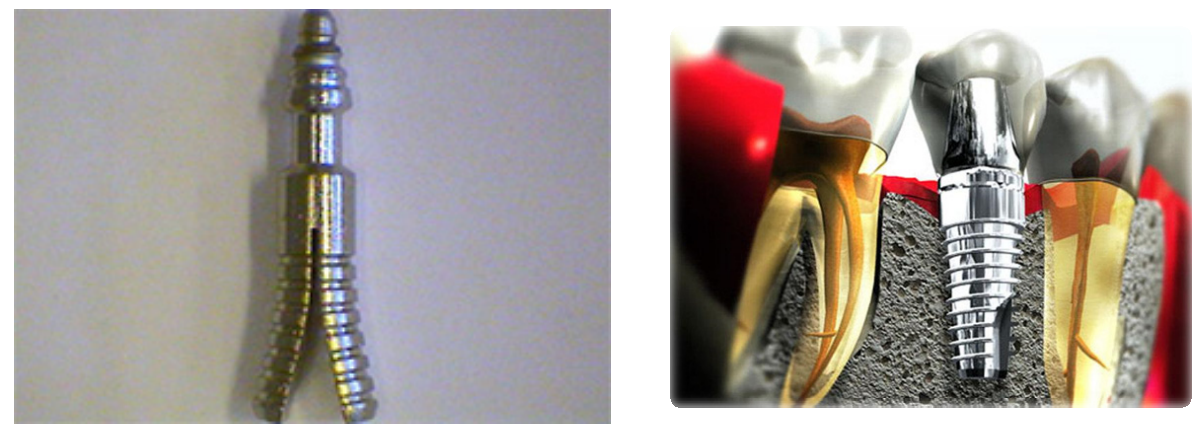

Рис. 1. Зубной имплантат из титанового сплава [1]

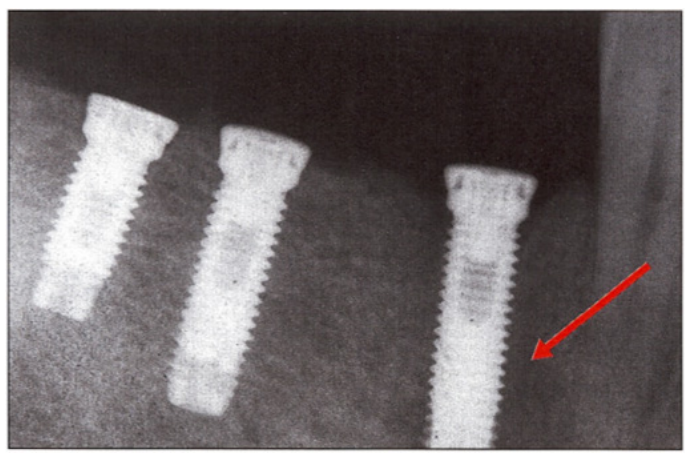

Рис. 2. Резорбция (разрушение) кости вокруг имплантата [5]

Наиболее удачное сочетание механических свойств имеется у сплавов системы титан - ниобий. Кроме того, ниобий и титан являются нетоксичными элементами и не вступают в реакцию с тканями организма. Некоторые сплавы системы титан - ниобий обладают низким модулем упругости.

Традиционные методы металлургии для получения титановых сплавов имеют ряд технических и принципиальных ограничений [6]. Имплантаты из титана и кобальтохромовых сплавов (КХС) изготавливают способом литья, фрезерования и токарной обработки, порошковой металлургии, горячей штамповки и штамповки с последующим фрезерованием. 
Литье является одним из способов изготовления имплантатов. Для расплавления стальных и кобальтохромовых сплавов применяются высокочастотные литейные установки, обеспечивающие хорошее качество отливок. Лабораторное изготовление имплантатов из сплавов КХС обычно не представляет трудностей. Отливка же имплантатов из титановых сплавов является более сложной по следующим причинам: при нагреве в воздухе титан взаимодействует с азотом и кислородом, а в расплавленном виде реагирует и с футеровкой формы. Технология литья обусловливает необходимость плавления сплава и заливки форм в вакууме, а также применение нетрадиционных высокоогнеупорных форм. В связи с этим изготовление имплантатов из титана с хорошим качеством поверхности достигается лишь в заводских условиях. Отлитые имплантаты проходят рентгеноструктурный контроль, при котором выявляют 5-8 \% брака [7].

Фрезерование пластиночных имплантатов оправдано только на стадии разработки новых конструкций из титана. При массовом производстве фрезеровка имплантатов из листа металла экономически не оправдана. Изготовление винтовых, цилиндрических имплантатов возможно в основном токарной обработкой.

Горячая штамповка является одним из основных способов обработки металлов, в том числе титана и КХС. Однако следует учитывать, что имплантаты - изделия малых объемов, а при нагреве газопоглощаемость увеличивается, поэтому установлены очень строгие требования к технологическому режиму. Оборудование для штамповки весьма дорогое и сложное, поэтому применение его обосновано только при изготовлении очень ответственных деталей в массовом объеме.

Штамповочно-фрезерный способ технологически более прост и при выпуске изделий большими партиями экономически обоснован. После попыток организовать изготовление титановых имплантатов выяснилось, что это самый доступный и приемлемый вариант. Имплантаты изготавливают в два этапа. На первом этапе специальным штампом из титанового листа толщиной 3 мм отрубают заготовки имплантатов. На втором этапе основа имплантата истончается на фрезерном станке одномоментно с двух сторон до 1,2 мм. После снятия заусениц имплантат готов к электрохимической полировке [7, 8].

На сегодняшний день способы послойного лазерного синтеза готовых изделий из различных порошковых материалов начинают успешно 
внедряться в одну из важнейших сфер деятельности - медицину. Это связано с более коротким циклом производства изделия-имплантата по сравнению с традиционными методами обработки (фрезерование, точение и т.п.), экономией дорогостоящих материалов, а также возможностью изготовления изделий сложной геометрии и заданной пористости $[9,10]$.

Принцип селективного лазерного сплавления (СЛС) заключается в формировании трехмерного изделия путем последовательного сплавления слоев порошкового материала лучом лазера по заданной программе (рис. 3).

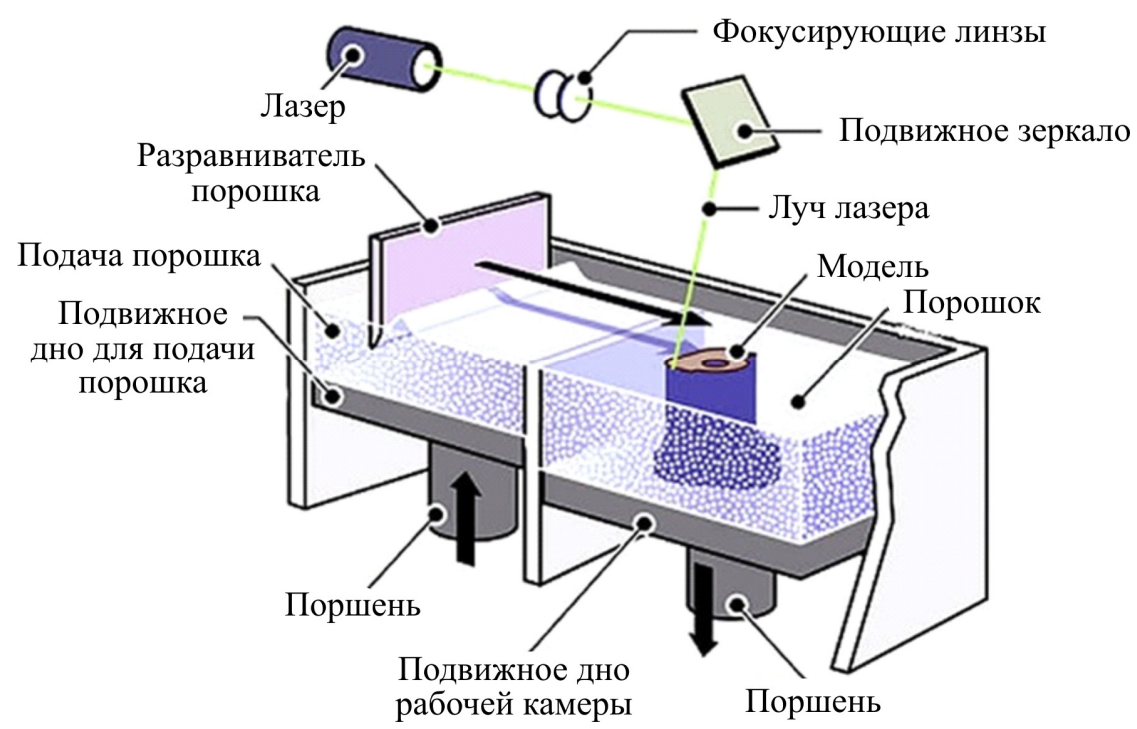

Рис. 3. Принципиальная схема СЛС [11]

Технология СЛС позволяет контролировать все основные технологические параметры, оказывающие влияние на процесс синтеза изделия, такие как мощность излучения, скорость сканирования, температура порошка [11-13]. Это дает возможность получать конечный продукт с заданными механическими свойствами [14].

Технологический процесс состоит из двух этапов: сначала ровный тонкий слой порошка равномерно размещается по всей рабочей площадке, после чего включается лазер и сплавляет области, которые соответствуют срезу создаваемого объекта. Затем поршень опускается вниз на расстояние, равное толщине слоя, и алгоритм повторяется, пока процесс не дойдет до самой верхней точки модели. 
На каждом этапе СЛС можно оптимизировать технологические режимы. Порошок может наноситься валиком либо ракелем. Сплавление может проводиться только на участке, который соответствует границе перехода (внешний контур слоя), или по всей глубине модели.

Важная особенность селективного лазерного сплавления - отсутствие необходимости в поддерживающих структурах, так как излишек окружающего порошка по всему объему не дает модели разрушиться, пока окончательная форма еще не обретена и не достигнута прочность конечного изделия [15].

Реализация СЛС композитного порошка в соотношении массовых долей 60 мас. \% Ті и 40 мас. \% Nb (Ti40Nb) выполнена на экспериментальной установке послойного лазерного спекания «ВАРИСКАФ-100МВ» [16]. Она предназначена для послойного синтеза изделий различной пространственной формы из порошкового материала методом селективного лазерного спекания/сплавления. Конструкция установки позволяет спекать изделия как в условиях вакуума, так и в различных газовых средах. Общий вид установки «ВАРИСКАФ-100МВ» представлен на рис. 4.

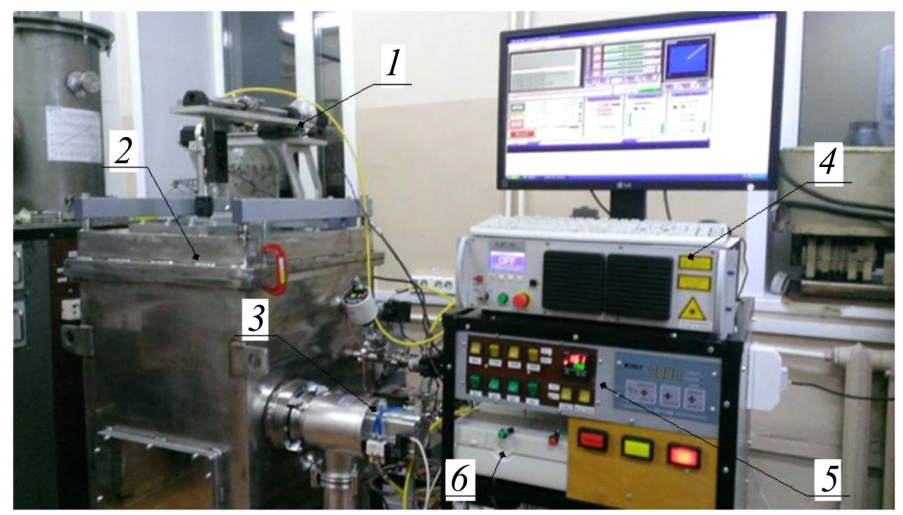

Рис. 4. Общий вид установки «ВАРИСКАФ-100МВ»: 1 - «летающая» оптика; 2 - вакуумная камера; 3 - система откачки; 4 - иттербиевый лазер; 5 - блок управления установкой; 6 - ЧПУ

В результате проведенных экспериментов была получена панель образцов при следующих технологических параметрах. Мощность излучения лазера составляла 68; 75; 86; 96 и 106 Вт; скорость сканирования варьировалась в диапазоне 0,017-0,05 м/с; шаг сканирования составлял 0,1 мм. 
Изготавливались монослойные образцы размером $10 \times 10$ мм при толщине слоя смеси порошка 0,7 мм. Образцы сплавлялись в среде аргона. В качестве подложки использовались пластины сплава ВТ1-0. Подложка с порошком нагревалась до $200{ }^{\circ} \mathrm{C}$ и выдерживалась в течение 15 мин в вакууме при заданной температуре, затем проводился процесс сплавления в аргоне. Температура процесса контролировалась термопарой типа ХА, спай которой находился в подложке. Аргон подавался в камеру на протяжении всего процесса сплавления.

На рис. 5 изображена рабочая зона, где происходит предварительный подогрев подложки и процесс сплавления.

Стратегия сканирования лазерным лучом - построчная зигзагом (рис. 6).

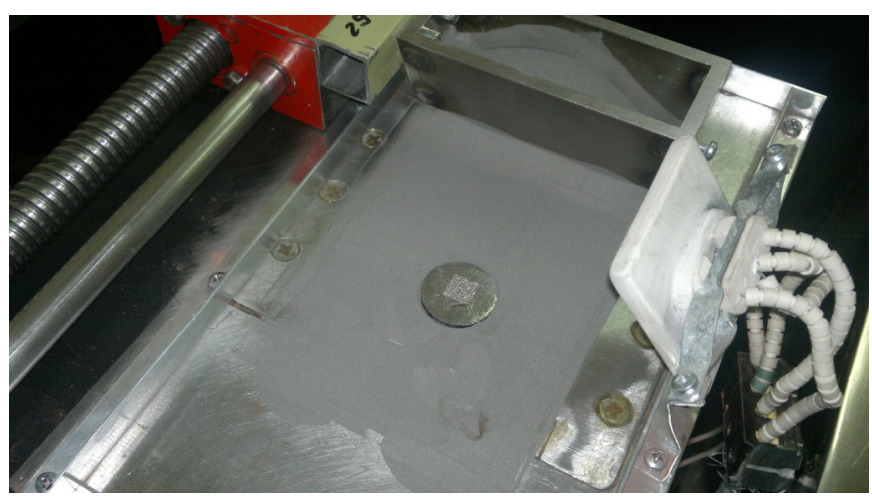

Рис. 5. Рабочая зона установки

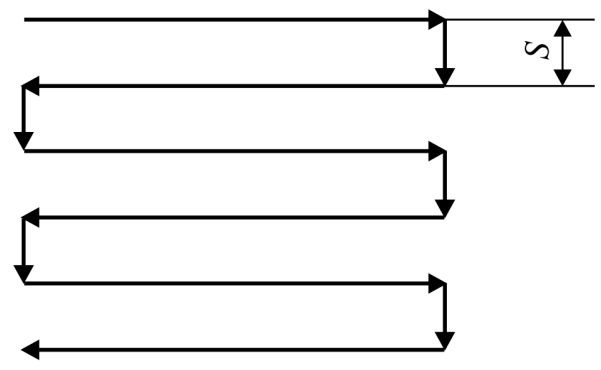

Рис. 6. Стратегия сканирования

Оптические изображения полученных образцов сплава Ti40Nb представлены в таблице. Хорошо видно, что при росте скорости сканирования увеличивается количество и размер пор. Образцы, полученные при мощности лазера 68-106 Вт и скоростях сканирования 0,05 и 0,042 м/с, визуально 
не имеют существенных отличий. Однако при уменьшении скорости сканирования до 0,017-0,042 м/с и увеличении мощности излучения до 96-106 Вт наблюдается уменьшении количества пор и образование равномерных участков расплавленного порошка.

\section{Одиночные слои сплава $\mathrm{Ti} 40 \mathrm{Nb}$}

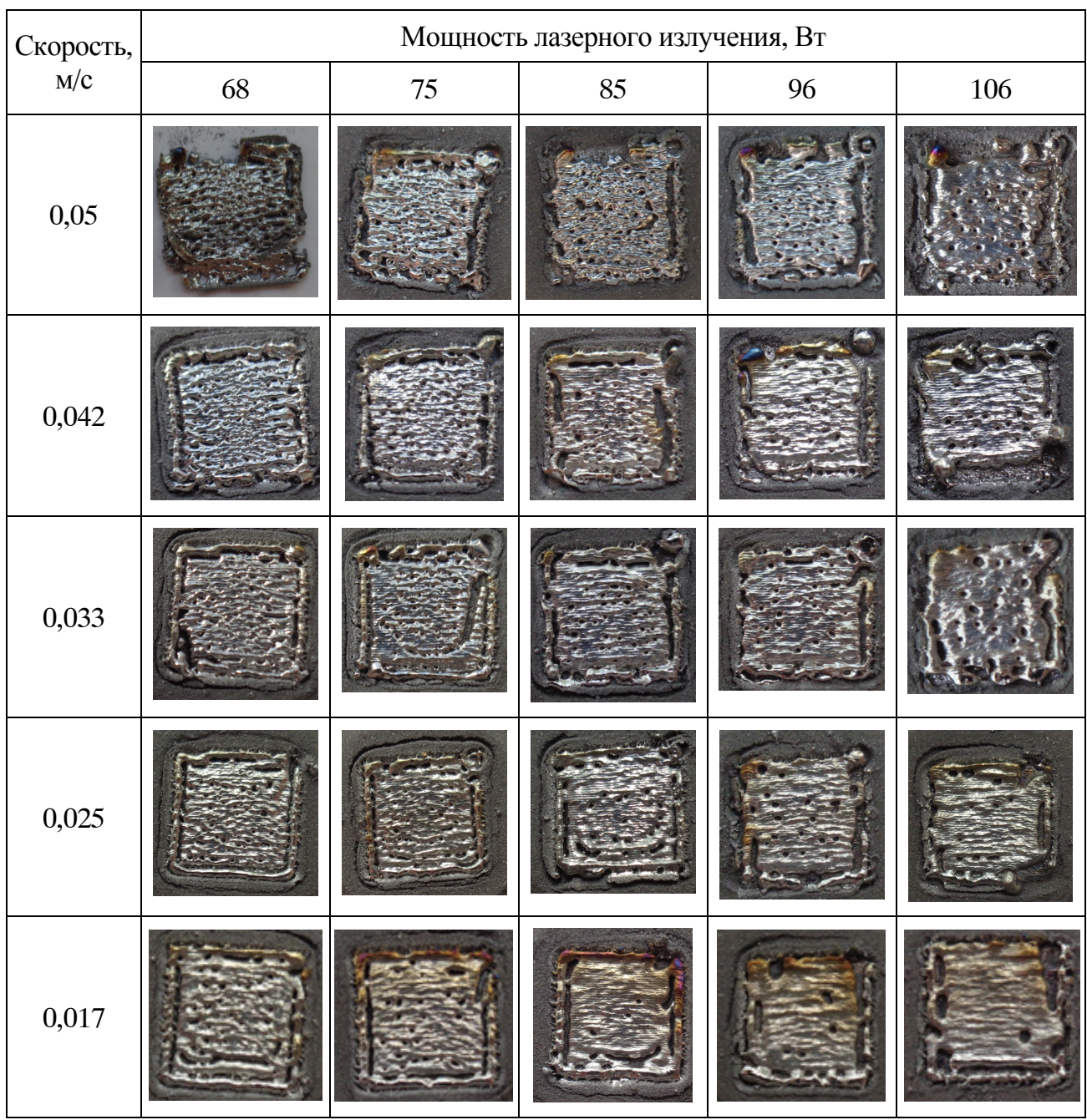

Увеличение мощности лазерного излучения и уменышение скорости сканирования сопровождается увеличением энерговклада лазерного излучения и ведет к уменьшению пористости, как показано на рис. 7. $\mathrm{C}$ понижением скорости сканирования увеличивается время воздействия 
лазерного излучения на локальный участок порошка, что приводит к более полному сплавлению слоя.

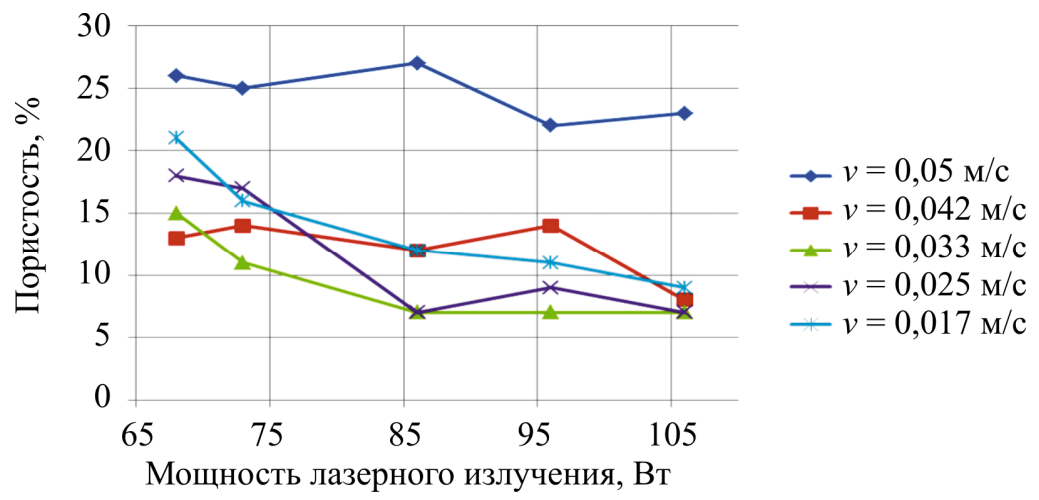

Рис. 7. Изменение пористости образцов от мощности лазерного излучения при разных скоростях сканирования

В образцах, полученных при мощности излучения 106 Вт, была измерена толщина сформированного слоя. Из рис. 8 видно, что с увеличением скорости сканирования глубина проплавления уменьшается.

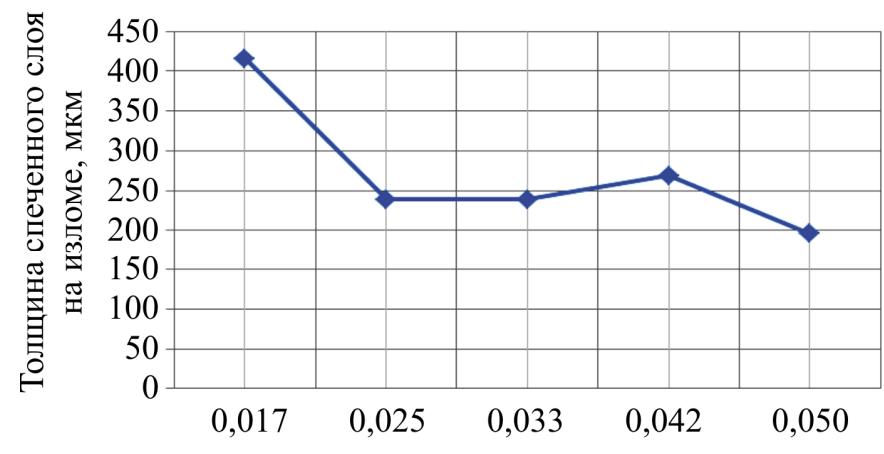

Скорость сканирования, м/с

Рис. 8. Изменение толщины сплавленного слоя образцов при изменении скорости сканирования

В связи с этим был проведен поисковый эксперимент и получен образец при мощности лазерного излучения 106 Вт, скорости сканирования 0,017 м/с и шаге сканирования 0,1 мм. Для увеличения энерговклада предварительный нагрев титановой подложки составил $400{ }^{\circ} \mathrm{C}$. Образец, полученный при максимальном энерговкладе для данной установки, представлен на рис. 9. 


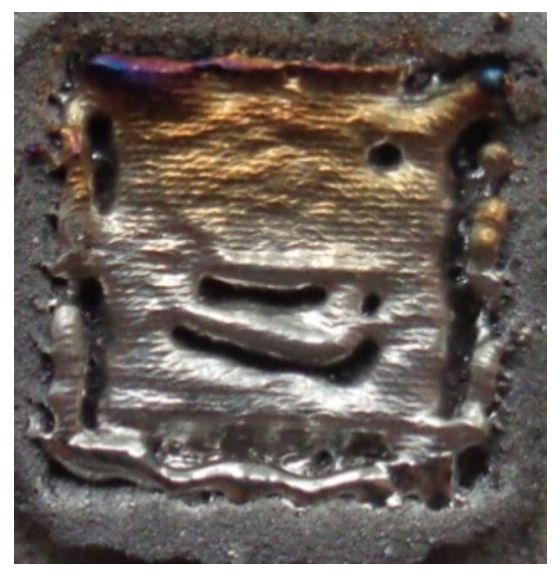

Рис. 9. Сплавленный слой композитного порошка при максимальном энерговкладе

Пористость образца составила около $9 \%$, однако значительно увеличилась толщина сплавленного слоя - до 570 мкм. Это можно объяснить увеличением температуры подогрева подложки и, как следствие, увеличением общего энерговклада.

Из проведенных исследований можно сделать вывод, что основными факторами, влияющими на формирование сплавленного слоя из композитного порошка $\mathrm{Ti} 40 \mathrm{Nb}$, являются мощность лазерного излучения и скорость сканирования. Необходимо отметить, что с уменьшением скорости сканирования и увеличением мощности лазерного излучения наблюдается значительное уменьшение пористости и увеличение толщины сформированного слоя сплава.

Работа выполнена при финансовой поддержке Российского научного фонда (грант № 15-19-00191). Авторы выражают благодарность руководителю проекта РНФ 15-19-00191 Ю.П. Шаркееву за участие в работе и обсуждение результатов исследований.

\section{Список литературы}

1. Материал с эффектом памяти формы (ЭПФ) и поверхностными слоями с повышенной коррозионной стойкостью и биосовместимостью для изготовления внутрикостного имплантата [Электронный ресурс] // Сайт Ин-та физики прочности и материаловедения CO PAH. - URL: http://www.ispms.ru/ru/production/45 (дата обращения: 22.12.2015). 
2. Mechanical alloying of beta-type $\mathrm{Ti}-\mathrm{Nb}$ for biomedical applications / K. Zhuravleva, S. Scudino, M.S. Khoshkhoo, A. Gebert, M. Calin, L. Schultz, J. Eckert // Advanced Engineering Materials. - 2013. - № 4. P. 262-268.

3. Microstructure and mechanical properties of Ti alloy with $40 \% \mathrm{Nb}$ mass after severe plastic deformation / Y.P. Sharkeev, A.Y. Eroshenko, I.A. Glukhov, Q. Zhu, A.I. Tolmachev // Physical Mesomechanics of Multilevel Systems. - 2014. - Vol. 1623. - P. 567-570.

4. Investigation of the influence of $\mathrm{Ti}-\mathrm{Nb}$ alloy composition on the structure of the ingots produced by arc melting / Y.P. Sharkeev, Z.G. Kovalevskaya, Q. Zhu, M.A. Khimich, E.A. Parilov // Advanced Materials Research. - 2015. - Vol. 1085. - P. 307-311.

5. Индивидуальное протезирование с использованием имплантатов [Электронный ресурс] // PBLG-концепция изготовления постоянных реставраций с опорой на имплантаты для беззубых пациентов. - URL: http://dentaltechnic.info/index.php/implanty (дата обращения: 22.12.2015).

6. Кузнецов В.Е. Системы быстрого изготовления прототипов и их расширения // CAD/CAM/CAE Observer. - 2003. - № 4(13). - С. 2-7.

7. Индивидуальное протезирование с использованием имплантатов [Электронный ресурс] // Основные способы изготовления имплантатов. - URL: http://dentaltechnic.info/index.php/implanty/zubnoe-protezyrovanye-na-ymplantatakh/2836-tyehnologiya_izgotovlyeniya_implanta (дата обращения: 22.12.2015).

8. Gibson I.B., Rosen D.W., Stucker B. Additive manufacturing technologies. Rapid prototyping to direct digital manufacturing. - New York: Springer, 2009. $-459 \mathrm{p}$.

9. Шишковский И.В., Закиев. Е.С., Холпанов Л.П. Послойный синтез объемных изделий из нитрида титана методом СЛС // Физика и химия обработки материалов. - 2005. - № 3. - С. 71-78.

10. Saprykina N.A., Saprykin A.A. Engineering support for improving quality of layer-by-layer laser sintering // The 7th International Forum on Strategic Technology IFOST2012, September 18-21, 2012. - Tomsk: TPU Press, 2012. - P. 129-132.

11. Что такое 3D-принтер? [Электронный ресурс] // Селективное лазерное спекание (SLS). - URL: http://3dpr.ru/selektivnoe-lazernoe-spekanie-sls (дата обращения: 22.12.2015). 
12. Kumar S., Kruth J.-P. Composites by rapid prototyping technology // Materials \& Design. - 2009. - P. 1-23.

13. Ibragimov E.A., Saprykin A.A., Babakova E.V. Influence of laser beam machining strategy at SLS synthesis // Advanced Materials Research. - 2014 - Vol. 1040. - P. 764-767.

14. Direct selective laser sintering of iron-graphite powder mixture / K. Murali, A.N. Chatterjee, P. Saha, R. Palai, S. Kumar, S.K. Roy, P.K. Mishra, A.R. Choudhury // Journal of Materials Processing Technology. - 2003. Vol. 136, iss. 1-3. - P. 179-185.

15. SLS-печать или селективное лазерное спекание [Электронный pecypc] // База знаний 3Dwiki. - URL: 3dwiki.ru/sls-pechat-ili-selektivnoe-lazernoe-spekanie (дата обращения: 22.12.2015).

16. А Сравнение способов активации медного порошка ПМС-1 для синтеза изделий методом SLS / А.А. Сапрыкин, А.В. Градобоев, В.И. Яковлев, Е.А. Ибрагимов, Е.В. Бабакова // Обработка металлов. 2015. - № 3 (68). - С. 82-88.

\section{References}

1. Material s effektom pamiati formy (EPF) i poverkhnostnymi sloiami s povyshennoi korrozionnoi stoikost'iu i biosovmestimost'iu dlia izgotovleniia vnutrikostnogo implantata [Material with shape memory effect (SME) and surface layers with high corrosion resistance and biocompatibility for the manufacture of intraosseous implant]. Sait Instituta fiziki prochnosti $i$ materialovedeniia Sibirskogo otdeleniia Rossiiskoi akademii nauk, available at: http://www.ispms.ru/ru/production/45 (accessed 22 December 2015).

2. Zhuravleva K., Scudino S., Khoshkhoo M.S., Gebert A., Calin M., Schultz L., Eckert J. Mechanical alloying of beta- Type Ti-Nb for biomedical applications. Advanced Engineering Materials, 2013, no. 4, pp. 262-268.

3. Sharkeev Y.P., Eroshenko A.Y., Glukhov I.A., Zhu Q., Tolmachev A.I. Microstructure and mechanical properties of Ti alloy with $40 \%$ $\mathrm{Nb}$ mass after severe plastic deformation. Physical Mesomechanics of Multilevel Systems, 2014, vol. 1623, pp. 567-570.

4. Sharkeev Y.P., Kovalevskaya Z.G., Zhu Q., Khimich M.A., Parilov E.A. Investigation of the influence of Ti-Nb alloy composition on the structure of the ingots produced by arc melting. Advanced Materials Research, 2015, vol. 1085, pp. 307-311. 
5. Individual'noe protezirovanie $\mathrm{s}$ ispol'zovaniem implantatov [Individual prosthetics with implants]. PBLG-kontseptsiia izgotovleniia postoiannykh restavratsii s oporoi na implantaty dlia bezzubykh patsientov, available at: http://dentaltechnic.info/index.php/implanty (accessed 22 December 2015).

6. Kuznetsov V.E. Sistemy bystrogo izgotovleniia prototipov i ikh rasshireniia [Systems of rapid manufacturing of prototypes and their widening]. CAD/CAM/CAE Observer, 2003, no. 4, pp. 2-7.

7. Individual'noe protezirovanie $\mathrm{s}$ ispol'zovaniem implantatov [Individual prosthetics with implants]. Osnovnye sposoby izgotovleniia implantatov, available at: http://dentaltechnic.info/index.php/implanty/zubnoe-protezyrovanye-na-ymplantatakh/2836-tyehnologiya_izgotovlyeniya_implanta (accessed 22 December 2015).

8. Gibson I.B., Rosen D.W., Stucker B. Additive manufacturing technologies. Rapid prototyping to direct digital manufacturing. New York: Springer, 2009. $459 \mathrm{p}$.

9. Shishkovskii I.V., Zakiev E.S., Kholpanov L.P. Posloinyi sintez ob"emnykh izdelii iz nitrida titana metodom SLS [Layer by layer synthesis of bulk products from titanium nitride SLS]. Fizika i khimiia obrabotki materialov, 2005, no. 3, pp. 71-78.

10. Saprykina N.A., Saprykin A.A. Engineering support for improving quality of layer-by-layer laser sintering. The 7th International Forum on Strategic Technology IFOST2012, September 18-21, 2012. Tomsk: TPU Press, 2012, pp. 129-132.

11. Chto takoe 3D printer? [What is a 3D printer?]. Selektivnoe lazernoe spekanie (SLS), available at: http://3dpr.ru/selektivnoe-lazernoe-spekaniesls (accessed 22 December 2015).

12. Kumar S., Kruth J.-P. Composites by rapid prototyping technology. Materials \& Design, 2009, pp. 1-23.

13. Ibragimov E.A., Saprykin A.A., Babakova E.V. Influence of laser beam machining strategy at SLS synthesis. Advanced Materials Research, 2014, vol. 1040, pp. 764-767.

14. Murali K., Chatterjee A.N., Saha P., Palai R., Kumar S., Roy S.K., Mishra P.K., Choudhury A.R. Direct selective laser sintering of iron-graphite powder mixture. Journal of Materials Processing Technology, 2003, vol. 136, iss. $1-3$, pp. 179-185.

15. SLS-pechat' ili selektivnoe lazernoe spekanie [SLS-printing or selective laser melting]. Baza znanii 3Dwiki, available at: 3dwiki.ru/sls-pechatili-selektivnoe-lazernoe-spekanie (accessed 22 December 2015). 
16. Saprykin A.A., Gradoboev A.V., Iakovlev V.I., Ibragimov E.A., Babakova E.V. Sravnenie sposobov aktivatsii mednogo poroshka PMS-1 dlia sinteza izdelii metodom SLS [Comparison of activation technologies powder ECP-1 for the synthesis of products using SLS]. Obrabotka metallov, 2015, no. 3(68), pp. 82-88.

Получено 22.12.2015

\section{Об авторах}

Елена Владимировна Бабакова (Юрга, Россия) - ассистент кафедры «Металлургия черных металлов», аспирант Юргинского технологического института Национального исследовательского Томского политехнического университета; e-mail: babakova@tpu.ru.

Маргарита Андреевна Химич (Томск, Россия) - инженер Института физики прочности и материаловедения СО РАН, аспирант Национального исследовательского Томского политехнического университета; e-mail: makhimich@gmail.com.

Александр Александрович Сапрыкин (Юрга, Россия) - кандидат технических наук, доцент кафедры «Металлургия черных металлов» Юргинского технологического института Национального исследовательского Томского политехнического университета; e-mail: sapraa@tpu.ru.

Егор Артурович Ибрагимов (Юрга, Россия) - ассистент кафедры «Металлургия черных металлов» Юргинского технологического института Национального исследовательского Томского политехнического университета; e-mail: egor83@list.ru.

\section{About the authors}

Elena V. Babakova (Yurga, Russian Federation) - Assistant, Department "Metallurgy Ferrous Metals", Postgraduate Student, Yurga Institute of Technology of National Research Tomsk Polytechnic University; e-mail: babakova@tpu.ru.

Margarita A. Khimich (Tomsk, Russian Federation) - Engineer, Institute of Strength Physics and Materials Science of the Siberian Branch of the Russian Academy of Sciences, Postgraduate Student, Yurga Institute of 
Technology of National Research Tomsk State University; e-mail: makhimich@gmail.com.

Aleksandr A. Saprykin (Yurga, Russian Federation) - Ph. D. in Technical Sciences, Associate Professor, Department "Metallurgy Ferrous Metals", Yurga Institute of Technology of National Research Tomsk Polytechnic University; e-mail: sapraa@tpu.ru.

Egor A. Ibragimov (Yurga, Russian Federation) - Assistant, Department "Metallurgy Ferrous Metals", Yurga Institute of Technology of National Research Tomsk Polytechnic University; e-mail: egor83@list.ru. 